\title{
Four-index equations for gravitation and the gravitational energy-momentum tensor ${ }^{1}$
}

\author{
Zahid Zakir ${ }^{2}$
}

\begin{abstract}
A new treatment of the gravitational energy on the basis of 4-index gravitational equations is reviewed. The gravitational energy for the Schwarzschild field is considered.
\end{abstract}

PACS: 04.20.Cv, 04.20.Fy, 11.10.-z

Key words: gravitational energy, curvature tensor, vacuum energy

\section{Content}

Introduction

1. Four-index equations for the gravitational field.

2. The gravitational energy for the Schwarzschild field ..................................................... 21

References

\section{Introduction}

A covariant physical characteristics of the gravitational field is the Riemann curvature tensor, and it is natural that the problems with the energy-momentum of gravitation can be solved if we can express the gravitational energy in terms of this tensor.

In the papers [1-2] a new generalized 4-index version of the Einstein equations with the Riemann tensor has been formulated, and the local energy-momentum tensors for the system of gravitation field and matter, linearly depending on the curvature tensor, have been constructed as 4-index tensors.

In the present paper some consequences of this treatment, including the calculation of the gravitational energy for a mass point, will be presented.

\section{Four-index equations for the gravitational field}

In the standard Einstein-Gilbert gravitational action one can add to the Ricci tensor or to the Riemann tensor arbitrary functions (tensors) with zero contractions:

$$
R=g^{k m} R_{k m}=\frac{1}{2}\left(g^{k m} g^{i l}-g^{i m} g^{k l}\right)\left(R_{i k l m}-\kappa V_{i k l m}\right),
$$

where $\kappa=8 \pi k / c^{4}$, and $L_{m}$ is the matter Lagrangian, $V_{i k l m}$ has the same symmetry properties as $R_{i k l m}$, and $g^{i l} V_{i k l m}=0$.

So, we can start from the new action function:

\footnotetext{
${ }^{1}$ The preprint of the paper has been presented in 1999 (revised 2003): Zakir Z. arXiv:gr-qc/9906039.

${ }^{2}$ Centre for Theoretical Physics and Astrophyics, Tashkent, Uzbekistan; zahidzakir@ theor-phys.org
} 


$$
S=\frac{1}{2} \int d \Omega \sqrt{-g}\left[\frac{1}{2}\left(g^{k m} g^{i l}-g^{i m} g^{k l}\right)\left(-\frac{1}{\kappa} R_{i k l m}+V_{i k l m}\right)+L_{m}\right],
$$

which is fully equivalent to the standard Einstein-Gilbert action function. Then we obtain for the variation of the action function [1]:

$$
\delta S=-\frac{1}{2} \int d \Omega \sqrt{-g} \delta g^{k m} g^{i l}\left(G_{i k l m}-T_{i k l m}\right)=0,
$$

where $T_{i k l m}=V_{i k l m}+T_{i k l m}^{(m)}$ and:

$$
\begin{aligned}
& G_{i k l m}=\frac{1}{\kappa}\left[R_{i k l m}-\frac{1}{(d-1)(d-2)}\left(g_{i l} g_{k m}-g_{i m} g_{k l}\right) R\right], \\
& T_{i k l m}^{(m)}=\frac{1}{(d-2)}\left(g_{k m} T_{i l}-g_{k l} T_{i m}+g_{i l} T_{k m}-g_{i m} T_{k l}\right)- \\
& -\frac{1}{(d-1)(d-2)}\left(g_{i l} g_{k m}-g_{i m} g_{k l}\right) T
\end{aligned}
$$

Here $\quad d$ is the spacetime dimensionality, and $T_{i k l m}$ has the same structure as the Riemann tensor having the representation:

$$
\begin{aligned}
& R_{i k l m}=C_{i k l m}+\frac{1}{(d-2)}\left(g_{k m} R_{i l}-g_{k l} R_{i m}+g_{i l} R_{k m}-g_{i m} R_{k l}\right)- \\
& -\frac{1}{(d-1)(d-2)}\left(g_{i l} g_{k m}-g_{i m} g_{k l}\right) R,
\end{aligned}
$$

where $C_{i k l m}$ is the Weyl tensor with zero 2-index contraction $g^{i l} C_{i k l m}=0$. Thus, we obtain the equations:

$$
g^{i l}\left(G_{i k l m}-T_{i k l m}\right)=0 .
$$

In a general case the expression in the parenthesis is not equal to zero for the arbitrary $V_{i k l m}$ and we cannot simply exclude the contractional factor $g^{i l}$. However the tensor $V_{i k l m}$ has 10 independent components which is equal to the number of the Riemann tensor components in the vacuum $\left(T_{k m}^{(m)}=0\right.$ ) where it is reduced to the Weyl tensor. Therefore, if in this case we choose the $V_{i k l m}$ as equal to:

$$
\frac{1}{\kappa} G_{i k l m}=\frac{1}{\kappa} C_{i k l m}=V_{i k l m},
$$

the equations hold identically for the solutions of the Einstein equations. Thus, we may write the 4-index equations for the gravitational field as [1]:

$$
G_{i k l m}=T_{i k l m} \text {. }
$$

We see that $V_{i k l m}$ can be considered as the 4-index energy-momentum density tensor for the gravitational field. Although its 2-index contraction vanish, in the 4-index form it allows one to determine a nonzero, local and positive defined energy-momentum tensor for the gravitational field. 
The tensors $G_{i k l m}$ and $T_{i k l m}$ have the symmetry properties of the Riemann tensor and, therefore, we have 20 equations. The tensor $G_{i k l m}$ is a function of the metric tensor $g_{i k}$ which has 6 independent components. The tensor $T_{i k l m}^{(m)}$ has been combined from the ordinary energy-momentum tensor of the matter $T_{i k}$ and it has 4 independent functions (the energy density $\epsilon$ and 3 components of the velocity). These 10 functions obey to 10 Einstein equations. The tensor $V_{i k l m}$ gives additional 10 independent components.

So, we have 20 equations for 20 independent functions. If we take solutions of the Einstein equations for the metrics and $T_{i k}$, then we have the additional 10 equations for 10 components of $V_{i k l m}$. Therefore, the solutions of the Einstein equations exactly define all components of $V_{i k l m}$ and we can find $V_{i k l m}$ for the known standard metrics.

In the vacuum $T_{i k}=T=0, R_{i l}=R=0$ and we have the equations Eq. (8). We see that in the vacuum the tensor $V_{i k l m}$ plays the role of the source for the empty spacetime curvature $C_{i k l m}$.

The covariant derivatives of the 4-index tensors are also related as:

$$
G_{k l m ; i}^{i}=T_{k l m ; i}^{i}
$$

In the case $d=4$ we have:

$$
\begin{gathered}
G_{. k l m ; i}^{i}=T_{k m ; l}-T_{k l ; m}-\frac{1}{3}\left(g_{k m} T_{, l}-g_{k l} T_{, m}\right) \\
T_{k l m ; j}^{j(m)}=\frac{1}{2}\left[T_{k m ; l}-T_{k l ; m}-\frac{1}{3}\left(g_{k m} T_{; l}-g_{k l} T_{; m}\right)\right]=\frac{1}{2} G_{. k l m ; i}^{i} .
\end{gathered}
$$

Then we obtain the relationship:

$$
V_{k l m ; j}^{j}=G_{k l m ; j}^{j}-T_{k l m ; j}^{j(m)}=\frac{1}{2} G_{. k l m ; i}^{i} .
$$

and, therefore,

$$
V_{k l m ; j}^{j}=T_{k l m ; j}^{j(m)}
$$

In the vacuum, therefore, there are local conservation laws:

$$
G_{\cdot k l m ; j}^{j}=V_{k l m ; j}^{j}=0 .
$$

The integral energy-momentum tensor for the system of matter and gravitational field can be defined as:

$$
P_{l m}^{i}=\int d S_{k} T_{. . l m}^{i k}
$$

On the hypersurface $x^{0}=$ const we have:

$$
P_{. l m}^{k}=\int d^{3} x \sqrt{-g} T_{. . l m}^{0 k}=\int d^{3} x \sqrt{-g}\left(T_{. . l m}^{(m) 0 k}+V_{. . l m}^{0 k}\right) .
$$

The energy-momentum vector for matter can be obtained as: $P^{i}=P_{. k}^{i k}$. Finally, the 3index integral energy-momentum of the gravitational field can be defined as: 


$$
P_{. l m}^{(g) i}=\int d^{3} x \sqrt{-g} V_{. . m}^{0 i} .
$$

\section{The gravitational energy for the Schwarzschild field}

Let us consider the energy of the Schwarzschild field with the line element:

$$
d s^{2}=\left(1-\frac{r_{g}}{r}\right) d t^{2}-\frac{d r^{2}}{1-\frac{r_{g}}{r}}-r^{2}\left(d \vartheta^{2}+\sin ^{2} \vartheta d \varphi^{2}\right),
$$

where $r_{g}=2 G m$ is the gravitational radius, and the components of the metric are: $g_{22}=-r^{2}, g_{33}=-r^{2} \sin ^{2} \vartheta$, and:

$$
g_{00}=g_{11}^{-1}=1-\frac{r_{g}}{r} .
$$

We calculate the energy-momentum tensor:

$$
V_{l m}^{i k}=\frac{1}{\kappa} R_{l m}^{i k}
$$

for this solution of the Einstein equations. Nonzero components of the $V_{i k l m}=R_{i k l m} / \kappa$ with this metric are:

$$
\begin{gathered}
V_{0101}=\frac{r_{g}}{\kappa r^{3}}=-V(r) g_{00} g_{11}, \\
V_{0202}=-\frac{r_{g}\left(r-r_{g}\right)}{2 \kappa r^{2}}=\frac{1}{2} V(r) g_{00} g_{22}, \\
V_{0303}=-\frac{r_{g}\left(r-r_{g}\right)}{2 \kappa r^{2}} \sin ^{2} \vartheta=\frac{1}{2} V(r) g_{00} g_{33}, \\
V_{1212}=\frac{r_{g}}{2 \kappa\left(r-r_{g}\right)}=\frac{1}{2} V(r) g_{11} g_{22}, \\
V_{1313}=\frac{r_{g} \sin ^{2} \vartheta}{2 \kappa\left(r-r_{g}\right)}=\frac{1}{2} V(r) g_{11} g_{33}, \\
V_{2323}=-\frac{r_{g} r}{\kappa} \sin ^{2} \vartheta=-V(r) g_{22} g_{33},
\end{gathered}
$$

where

$$
V(r)=\frac{r_{g}}{\kappa r^{3}}=\frac{m}{4 \pi r^{3}}=-\frac{m}{8 \pi} \frac{\partial}{\partial r}\left(r^{-2}\right) .
$$

We see that the 2-index contraction of this tensor vanishes:

$$
V_{i l}=g^{k m} V_{i k l m}=g_{i l}\left[-V(r)+\frac{1}{2} V(r)+\frac{1}{2} V(r)\right]=0 .
$$

The physical components of the gravitational energy-momentum tensor $V_{. . l m}^{i k}=g^{i p} g^{k q} V_{p q l m}$ are:

$$
V_{. .01}^{01}=V_{. .10}^{10}=V_{. .23}^{23}=V_{. .32}^{32}=-V(r),
$$


22 Zakir Z. (2010) Theoretical Physics, Astrophysics and Cosmology, 5, 22; TPAC: 3600-019 v2.

$$
\begin{aligned}
& V_{. .02}^{02}=V_{. .20}^{20}=V_{. .03}^{03}=V_{. .30}^{30}=V_{. .12}^{12}= \\
& =V_{. .21}^{21}=V_{. .13}^{13}=V_{. .31}^{31}=\frac{1}{2} V(r) .
\end{aligned}
$$

They allow us to calculate one of components of integral gravitational energy-momentum around the static spherical source as:

$$
c P_{.01}^{(g) 1}=\int d S_{0} \sqrt{-g} V_{. .01}^{01}=\int d S_{0} \sqrt{-g}[-V(r)] .
$$

The spatial volume integral can be represented as a spatial surface integral and we obtain:

$$
c P_{.01}^{(g) 1}=\frac{m}{8 \pi} \int d S_{0} \sqrt{-g} \frac{\partial}{\partial r}\left(r^{-2}\right)=\frac{m}{8 \pi} \int d f_{0 r} r^{-2}=\frac{1}{2} n_{r} m,
$$

where $d f_{0 r}=n_{r} r^{2} d o$ is 2-dimensional surface element with the normal vector $n_{r}$ directed along $r$.

\section{References}

1. Zakir Z. (2010) Theor. Phys., Astrophys. \& Cosmol., 5, 2, 14; doi:

New equations for gravitation with Riemann tensor and 4-Index energy-momentum tensors for gravitation and matter, arXiv:gr-qc/9905009, 4 p.

2. Zakir Z. (2010) Theor. Phys., Astrophys. \& Cosmol., 5, 2, 18; Four-index energy-momentum tensors for gravitation and matter, arXiv:gr-qc/9905036, 4 p. 